\title{
In Situ Observation of the Dislocation Structure Evolution During a Strain Path Change in Copper
}

\author{
CHRISTIAN WEJDEMANN,${ }^{1}$ HENNING FRIIS POULSEN,${ }^{2}$ \\ ULRICH LIENERT ${ }^{3}$ and WOLFGANG PANTLEON ${ }^{1,4}$
}

\begin{abstract}
1.-Department of Mechanical Engineering, Technical University of Denmark, 2800 Kgs. Lyngby, Denmark. 2.-Department of Physics, Technical University of Denmark, 2800 Kgs. Lyngby, Denmark. 3.-DESY Photon Science, Deutsches Elektronen-Synchrotron, 22603 Hamburg, Germany. 4.-e-mail: pawo@dtu.dk
\end{abstract}

\begin{abstract}
The evolution of deformation structures in individual grains embedded in polycrystalline copper specimens during strain path changes is observed in situ by high-resolution reciprocal space mapping with high-energy synchrotron radiation. A large number of individual subgrains is resolved; their behavior during the strain path change is revealed and complemented by the analysis of radial x-ray peak profiles for the entire grain. This allows distinction between two different regimes during the mechanically transient behavior following the strain path change: Below $0.3 \%$ strain, the number and orientation of the resolved subgrains change only slightly, while their elastic stresses are significantly altered. This indicates the existence of a microplastic regime during which only the subgrains deform plastically and no yielding of the dislocation walls occurs. After reloading above $0.3 \%$ strain, the elastic stresses of individual subgrains are about the same as in unidirectionally deformed reference specimens. They increase only slightly during further straining-accompanied by occasional emergence of new subgrains, abundant orientation changes, and disappearance of existing subgrains.
\end{abstract}

\section{INTRODUCTION}

During plastic deformation of metals, dislocations are stored in the material. In pure face-centered cubic metals, they organize themselves into heterogeneous structures characteristic of the material and deformation conditions. Understanding the origin and evolution of the ordered dislocation structures is relevant for predicting a variety of mechanical properties such as flow stress and workhardening rates. ${ }^{1}$

The dislocation structures formed during plastic deformation of copper have been studied extensively with electron microscopy, e.g., Refs. ${ }^{2-6}$, and by x-ray line profile analysis, e.g., Refs. ${ }^{7-11}$. From such studies it is known that (I) the dislocations organize in ordered structures consisting of almost dislocation-free regions (subgrains) separated by dislocation walls, (II) the scale of the structure decreases inversely with increasing flow stress, ${ }^{12}$ and (III) the specific morphology depends on the deformation conditions and the orientation of the crystalline lattice. ${ }^{6}$
Nevertheless, our understanding of the interaction between subgrains and their evolution during plastic deformation is still rudimentary, in part due to the fact that none of the above techniques are able to visualize the embedded subgrains nondestructively.

Within the last decade, two synchrotron radiation-based techniques have been established to overcome this limitation:

- Differential aperture $x$-ray microscopy. This technique is based on scanning the surface of a sample with respect to a submicronsized x-ray beam. ${ }^{13}$ Depth resolution is provided by a triangulation principle involving incremental translations of an absorbing platinum wire through the diffracted beams. For each point (or voxel) in real space, the average elastic strain is derived. This technique has been used to study the distribution of elastic strains in $\mathrm{Cu}$ single crystals deformed by about $30 \%$. The achieved spatial resolution $0.5 \mu \mathrm{m} \times$ $0.5 \mu \mathrm{m} \times 0.5 \mu \mathrm{m}^{14}$ (which has been improved slightly to $\left.0.33 \mu \mathrm{m} \times 0.46 \mu \mathrm{m} \times 0.5 \mu \mathrm{m}^{15}\right)$ is sufficient to 
map the dislocation structure of such moderately deformed specimens in three dimensions. While quite comprehensive results are obtained, this method has - to the knowledge of the authors-not been used in connection with in situ deformation studies due to geometric constraints and the low speed of data acquisition.

- High-resolution reciprocal space mapping. By this method, reciprocal space maps are provided of single grains embedded in bulk specimens. ${ }^{16,17}$ Direct space information on the position of subgrains and neighbor relationships is not available, but the angular resolution is sufficient to allow identification of sharp peaks arising from subgrains; in this manner, information can be gathered on the size, orientation, and strain of individual subgrains. Furthermore, reciprocal space maps can be acquired sufficiently fast that in situ studies can be performed during loading.

In this article, we pursue high-resolution reciprocal space mapping with a focus on small strain increments in order to be able to follow the evolution of the subgrain structure in situ.

Furthermore, we study the effect of a tensiontension strain path change on the evolution of individual subgrains. Such changes are known to give rise to reorganization of the dislocation structure and are associated with a transient mechanical behavior of significant interest for metal forming processes. ${ }^{18,19}$ Our focus here is on how resilient the individual subgrains are toward the new deformation conditions and to which extend the reorganization occurs collectively.

\section{EXPERIMENTAL}

\section{Samples}

The sample material was $99.9 \%$ pure oxygen-free high-conductivity $\mathrm{Cu}$. The material had been annealed at $550^{\circ} \mathrm{C}$ for $2 \mathrm{~h}$, then cold-rolled in nine passes to a total reduction of $85 \%$, and subsequently annealed in vacuum at $450^{\circ} \mathrm{C}$ for another $2 \mathrm{~h}$. Characterization by electron backscatter diffraction showed that the material processed in this manner was fully recrystallized with a mean grain size of approximately $20 \mu \mathrm{m}$ and a texture close to random. A significant fraction of the grains was twinned. Finally, the material was predeformed at room temperature in uniaxial tension to a plastic strain of $5 \%$, with the predeformation axis parallel to the original rolling axis.

Tensile samples were spark cut from the predeformed material in two different shapes: dog-boned samples with a gauge length of $8 \mathrm{~mm}$ and a gauge width of $3 \mathrm{~mm}$ for the x-ray experiments, and rectangular samples with a length of $48 \mathrm{~mm}$ and a width of $8 \mathrm{~mm}$ for complementary mechanical tests. The thickness of all samples was $0.32 \mathrm{~mm}$. The angle between the long axis of these specimens (the final axis for tensile deformation) and the tensile axis of the predeformation was varied. In the following we report on samples with angles of $0^{\circ}$ (second deformation axis identical to predeformation axis) and $55^{\circ}$. The latter corresponds to a so-called orthogonal strain path change, where the ratio between the reloading yield stress and the stress in monotonic straining is the largest. ${ }^{18}$

\section{Mechanical Testing}

The results of conventional tensile tests with a strain rate of approximately $10^{-4} \mathrm{~s}^{-1}$ with and without a strain path change are shown in Fig. 1 . The reloading stress after strain path change is larger than the stress required during monotonic loading. For monotonic deformation, a linear decrease of the work-hardening rate with the flow stress is observed, whereas the specimens objected to the strain path change initially show a high workhardening rate decreasing rapidly over a very short strain interval followed by a linear decrease of the work-hardening rate with a different slope. Consequently, differences in the mechanical behavior are not restricted to a short transient, but persist to larger strains. ${ }^{20}$

\section{Experimental Methodology}

The experimental setup at beam line 1-ID at the Advanced Photon Source (APS) is described in detail in Refs. ${ }^{16}$ and ${ }^{17}$. The unique optics ${ }^{21}$ at the beam line provides a monochromatic beam (of $52 \mathrm{keV}$ ), which is both very penetrating and associated with favorable specifications such as a low bandwidth $\left(\Delta E / E=7 \times 10^{-5}\right)$, a small divergence $(11 \mu \mathrm{rad}$ horizontally and $17 \mu \mathrm{rad}$ vertically), and dimensions comparable to the average grain size $(25 \mu \mathrm{m} \times 30 \mu \mathrm{m})$. The sample was mounted in a small load frame enabling tensile deformation placed in an Eulerian cradle. The diffracted beam was transmitted through the sample and detected at one of 2 two-dimensional detectors.

The first detector was a $2048 \times 2048$ pixel detector with a pixel size of $200 \mu \mathrm{m}$ placed at a distance of $680 \mathrm{~mm}$ from the sample, implying that all DebyeScherrer rings up to and including the 400 ring were fully observable. This detector was used to identify suitable grains and goniometer settings, characterized by five criteria:

1. The grain is positioned close to the center axis of the specimen and therefore exhibits a dynamics which is representative of bulk behavior.

2. The grain is small enough to be completely illuminated by the beam.

3. The grain has a 400 reflection with a diffraction vector nearly parallel to the tensile direction.

4. This reflection is clearly separated in reciprocal space from any reflections from any of the other grains simultaneously illuminated.

5. The grain does not contain a twin. 

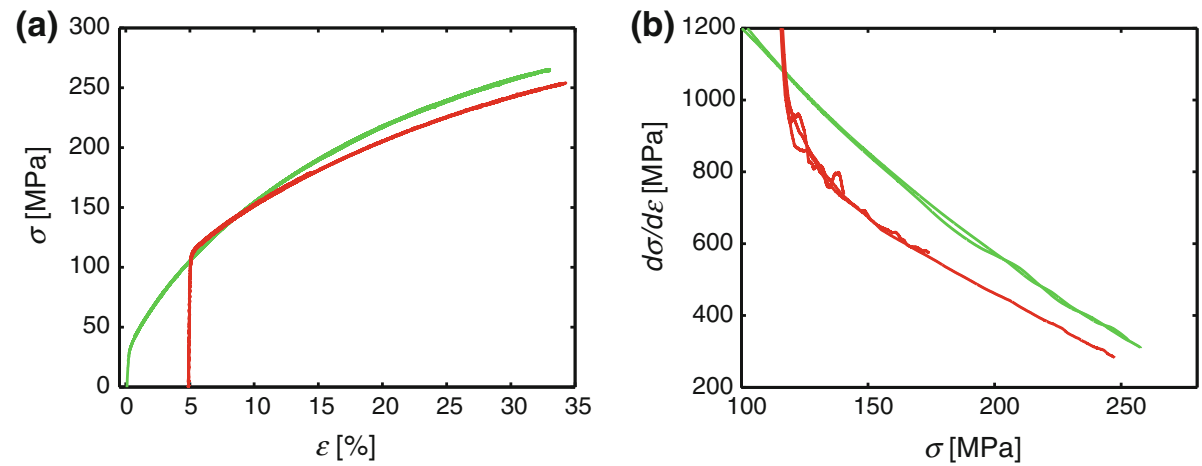

Fig. 1. Mechanical behavior: (a) true stress-true strain curves for samples deformed in tension along an axis making an angle of $55^{\circ}$ with the rolling direction and (b) corresponding work-hardening rates versus true stress. The green curves correspond to samples deformed without any strain path change. The red curves correspond to an orthogonal strain path change with an angle of $55^{\circ}$ between the tensile directions.

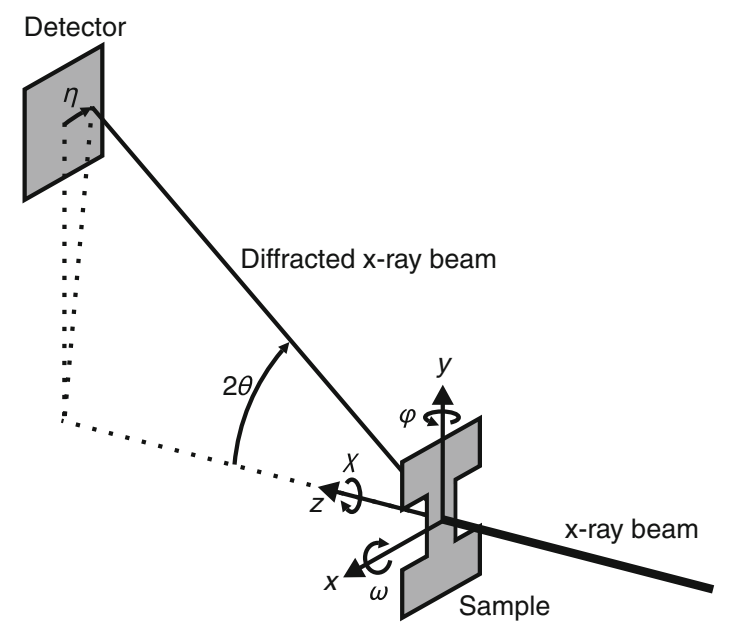

Fig. 2. Sketch showing the various coordinates and the diffraction geometry when the far detector is used.

Once such a grain and an associated 400 reflection had been found, this detector was translated out of the beam and the in situ loading experiment was performed with a $2048 \times 2048$ pixel detector with a pixel size of $79 \mu \mathrm{m}$ placed at the much larger distance of $3650 \mathrm{~mm}$ from the sample (cf. the geometry in Fig. 2). This second detector provided a zoom image of the 400 reflection of interest. By rotating (rocking) the sample in equiangular steps around a rotation axis perpendicular to the vertical scattering plane, a series of such images was obtained. When stacked, this set of images comprise a threedimensional (3-D) reciprocal space map of the 400 reflection in question. ${ }^{17}$ With an angular step size of $0.007^{\circ}$, the resolution of the map is nearly identical in all three directions of reciprocal space, namely $9 \times 10^{-4} \AA^{-1}$. Depending on the scattering angle $2 \theta$ and the $\mathrm{x}$-ray wave length $\lambda$, the following coordinates are used for describing reciprocal space:

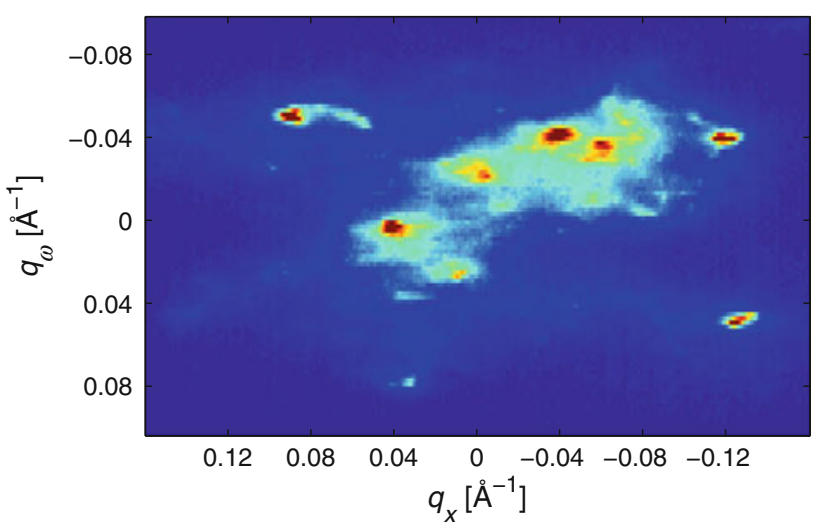

Fig. 3. Example of an azimuthal map of a reflection from a grain embedded in a sample predeformed to a tensile strain of $5 \%$ (minor elastic reloading to a strain of $0.03 \%$ is required to mount the specimen in the load frame).

$$
\begin{aligned}
q & =\frac{4 \pi}{\lambda} \sin \theta \\
q_{x} & =-\left(\eta-\eta_{0}\right) \frac{2 \pi}{\lambda} \sin (2 \theta) \\
q_{\omega} & =\left(\omega-\omega_{0}\right) \frac{4 \pi}{\lambda} \sin \theta
\end{aligned}
$$

An example of such a reciprocal space map for a grain from a specimen predeformed to 5\% is shown in Fig. 3. For ease of visualization, the 3-D map has been projected along the radial direction to produce a so-called azimuthal map-representing a zoom of the 400 pole figure with high magnification for the grain of interest. The map is comprised of a set of local intensity maxima on a smoothly varying contribution. We have demonstrated previously ${ }^{16,17,22}$ that these peaks correspond to the response from individual dislocation-free subgrains (or the 

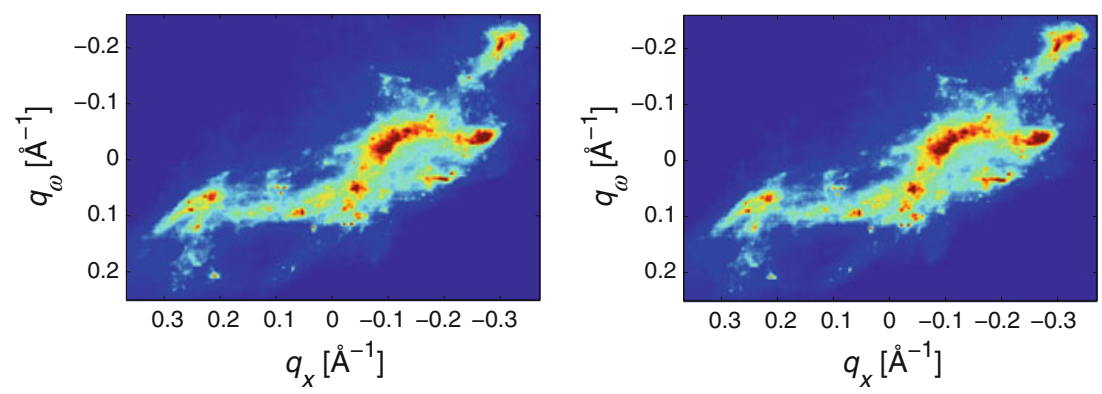

Fig. 4. Stability test. Two azimuthal maps of a grain embedded in a sample predeformed to a tensile strain of $5 \%$ obtained with a $10 \mathrm{~h}$ time difference.

superposition of such contributions) with volumes of 1 to $2 \mu \mathrm{m}^{3}$. The dislocation walls on the other hand give rise to more diffuse features; they are responsible for the slowly varying contribution of lower intensity in the map.

By means of the load frame, the samples were deformed in uniaxial tension with a strain rate of approximately $10^{-4} \mathrm{~s}^{-1}$. During the in situ deformation, strain and applied load were continuously measured by a strain gauge and a load cell, respectively. At a number of different strain levels, the deformation was interrupted. At each such step, the selected grain and the reflection of interest were recentered in the beam and on the detector by small changes in the settings of the goniometer, and a new reciprocal space map was acquired. The acquisition time for each image was $20 \mathrm{~s}$. With typically 140 angular steps, each reciprocal space map took approximately $1 \mathrm{~h}$ to acquire.

The simultaneously recorded stress-strain curves indicated a drop in the load by a few percent during each interruption. Past experiments have shown that this stress relaxation does not significantly alter the morphology of the dislocation structures. ${ }^{23}$

To further test the long-term stability of the setup and the reproducibility of the results, one sample predeformed to 5\% was mounted and left unaltered on the instrument for $10 \mathrm{~h}$. During this period, eight reciprocal space maps were obtained from one grain at regular intervals during a period of $10 \mathrm{~h}$. After normalization, the eight maps are virtually identical, as documented by the azimuthal maps for the first and last reciprocal space map, shown in Fig. 4. Evidently, to a very good approximation, the subgrain morphology and orientations are fixed during the $10 \mathrm{~h}$. This correspondence proofs at the same time the excellent stability of the setup.

\section{Data Analysis}

The intensity in the images was partitioned by a bicubic spline fit into two contributions: a smoothly varying cloud representing the contribution from the dislocation walls and a high-frequency component representing all the subgrains. ${ }^{24}$ Individual sharp peaks were identified by searching for local maxima in the subgrain component. The maxima found were subjected to a filter that removed peaks which were narrower than the instrumental resolution (the cutoff was set at $1.3 \times 10^{-3} \AA^{-1}$ ). The eighty most intense sharp peaks were kept for further analysis. It should be emphasized that some of these sharp peaks may represent the overlap of contributions from several subgrains diffracting in (almost) the same direction.

The position, width, and height of the sharp highintensity peaks were determined in all three directions of the reciprocal space by fitting of a split pseudo-Voigt profile. The uncertainty in the positions and widths is estimated to be less than $5 \times 10^{-4} \AA^{-1}$.

For details of other aspects of the data analysis (background subtraction, conversion to reciprocal space coordinates, and determination of the 3-D position of the grain), see Ref. 25.

\section{RESULTS}

\section{Unidirectional Loading (Without Strain Path Change)}

Figure 5 presents eight azimuthal maps obtained at several strain steps from $0.03 \%$ to $1.03 \%$ during reloading of a specimen in tension along the same direction as the tensile direction of the predeformation. Already in the unloaded state, the characteristic feature of high-intensity peaks on a cloud of enhanced intensity is obvious. An inspection of the maps obtained at the subsequent strain steps reveals that only minor changes occur in the azimuthal maps for strains below $0.3 \%$, whereas for strains above $0.3 \%$, changes can be identified clearly and the structure evolves gradually. During the initial regime, the number and orientations of the subgrains are conserved, whereas for strains above $0.3 \%$ splitting of existing and occasionally emergence of new peaks can be detected corresponding to a refinement of the subgrain structure.

\section{Orthogonal Strain Path Change}

In a similar manner, eight azimuthal maps of the loading behavior after a strain path change for strain steps from $0.03 \%$ to $0.93 \%$ are presented in Fig. 6. In this case, the new tensile direction forms an angle of 

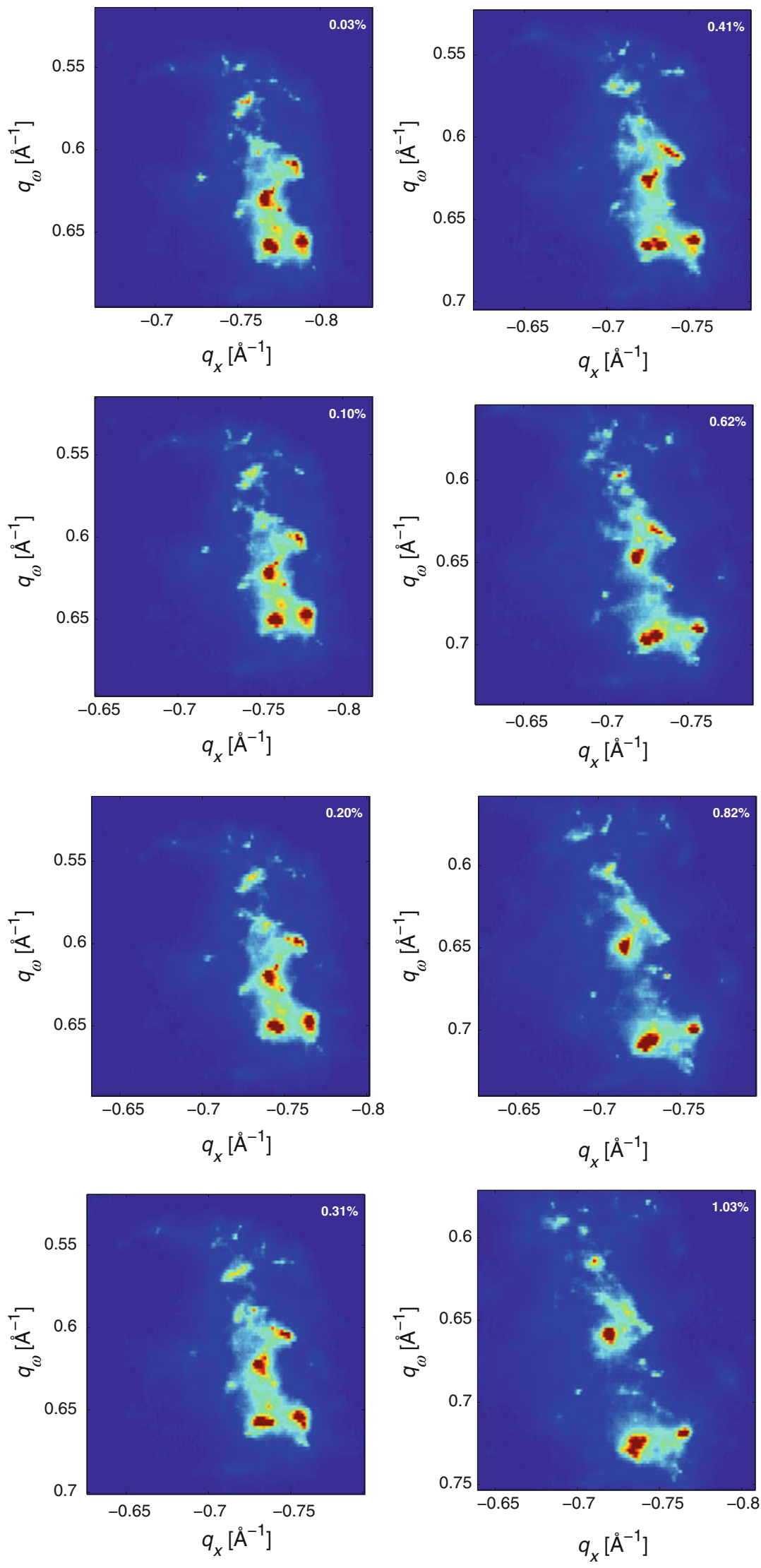

Fig. 5. Eight azimuthal maps from a grain embedded in a sample predeformed to a tensile strain of $5 \%$ obtained during in situ tensile deformation along the same axis as the predeformation (unidirectional deformation without strain path change). The strain levels indicated refer to the macroscopic strain following the $5 \%$ prestrain; for each map, the color scale is adjusted to the maximum intensity. 

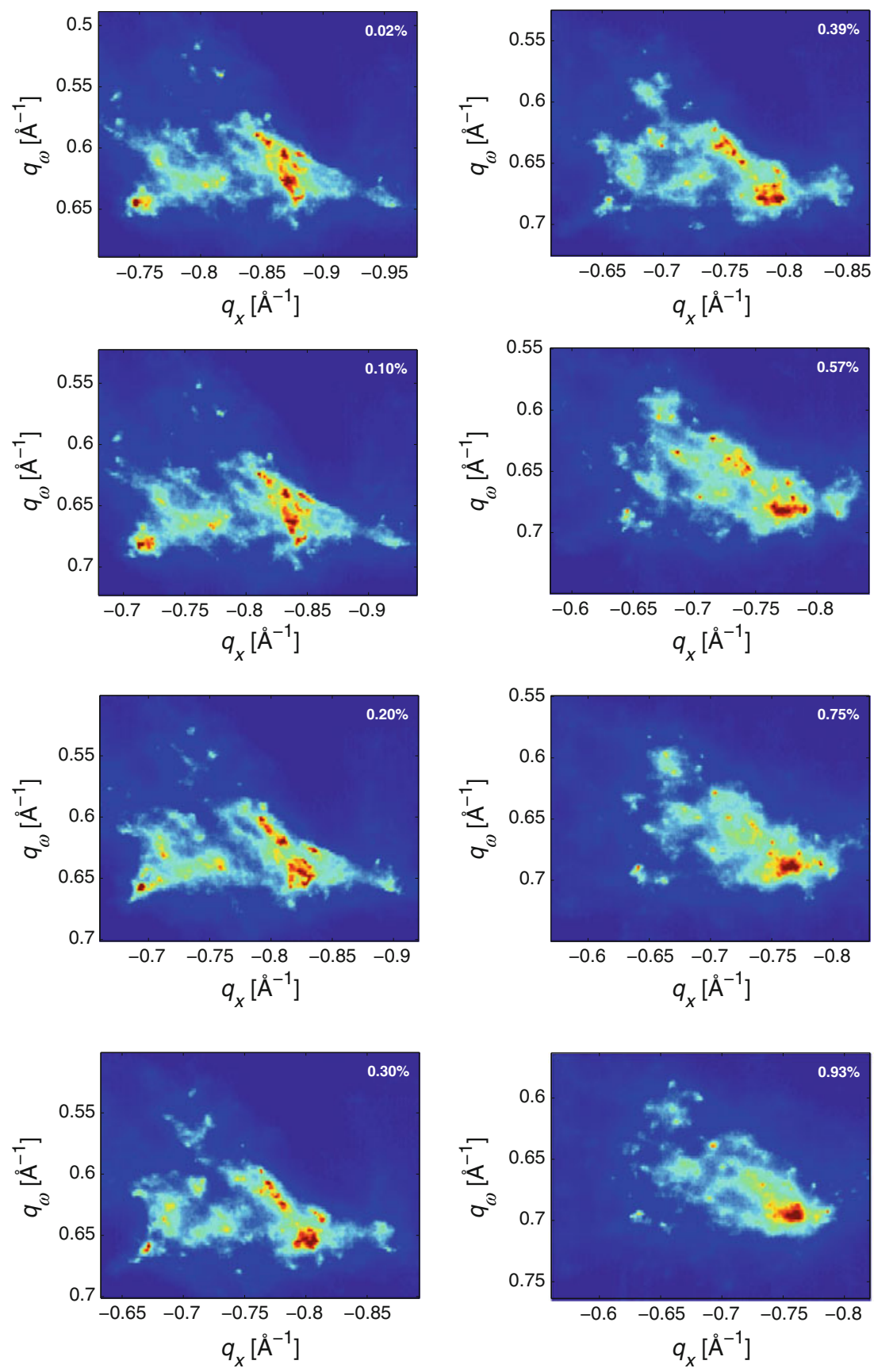

Fig. 6. Eight full azimuthal maps from a grain embedded in a sample predeformed to a tensile strain of $5 \%$ obtained during in situ tensile deformation after an orthogonal strain path change $\left(55^{\circ}\right.$ between the tensile axes). The strain levels indicated refer to the macroscopic strain following the $5 \%$ prestrain; for each map, the color scale is adjusted to the maximum intensity.

$55^{\circ}$ with the tensile direction of the predeformation. Again two regimes can be distinguished: Up to a strain of $0.2 \%$ to $0.3 \%$, the features of the azimuthal map are conserved and the high-intensity peaks remain at about the same azimuthal position. For strains larger than $0.3 \%$, their number increases and the azimuthal positions change more strongly.

The initial regime, during which the azimuthal maps are essentially preserved and neither the number of high-intensity peaks nor their azimuthal positions are altered significantly, is identified as a microplastic regime. During this transient regime, no new subgrains are formed. Following this initial regime, the subdivision and reorientation of existing subgrains and the occasional formation of new smaller subgrains becomes evident.

As an example of the structural changes in the later regime, partial azimuthal maps are presented for strains from $1.08 \%$ in even smaller strain increments of $0.03 \%$. Figure 7 makes it possible to 

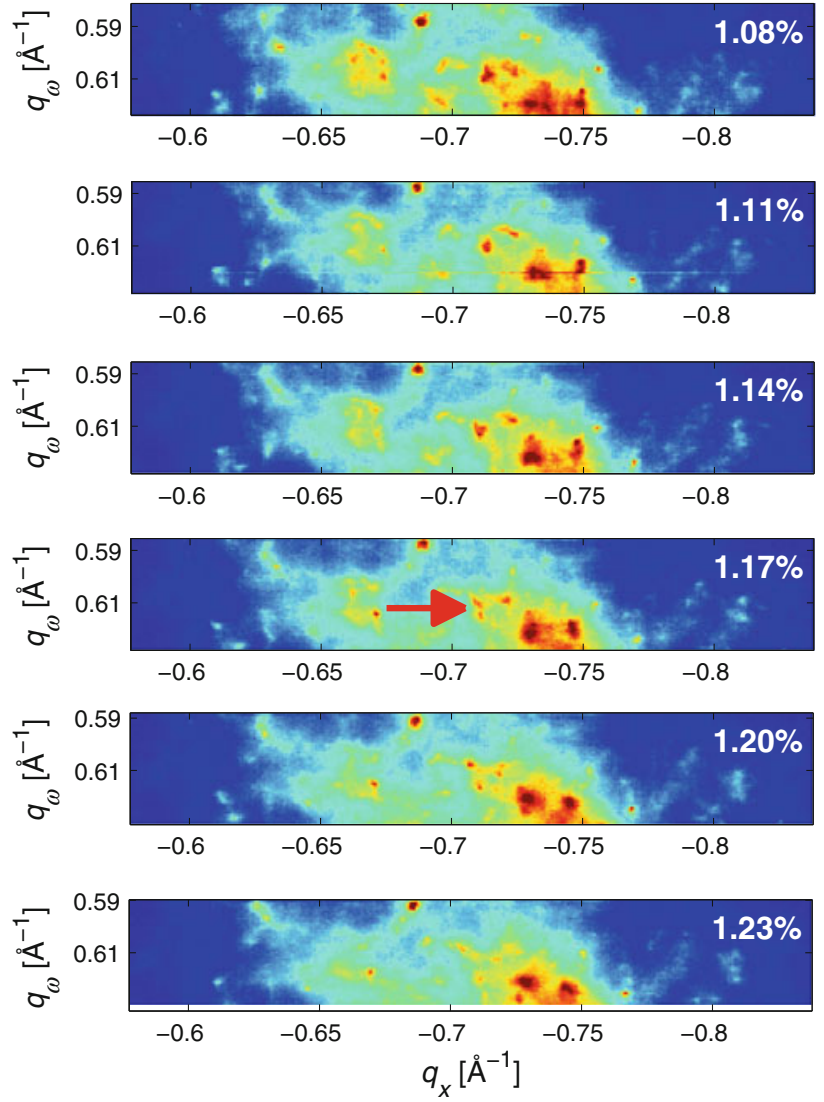

Fig. 7. Six partial azimuthal maps from a grain embedded in a sample predeformed to a tensile strain of $5 \%$ obtained during in situ tensile deformation after an orthogonal strain path change $\left(55^{\circ}\right.$ between the tensile axes). The strain levels indicated refer to the macroscopic strain following the $5 \%$ prestrain; for each map, the color scale is adjusted to the maximum intensity.

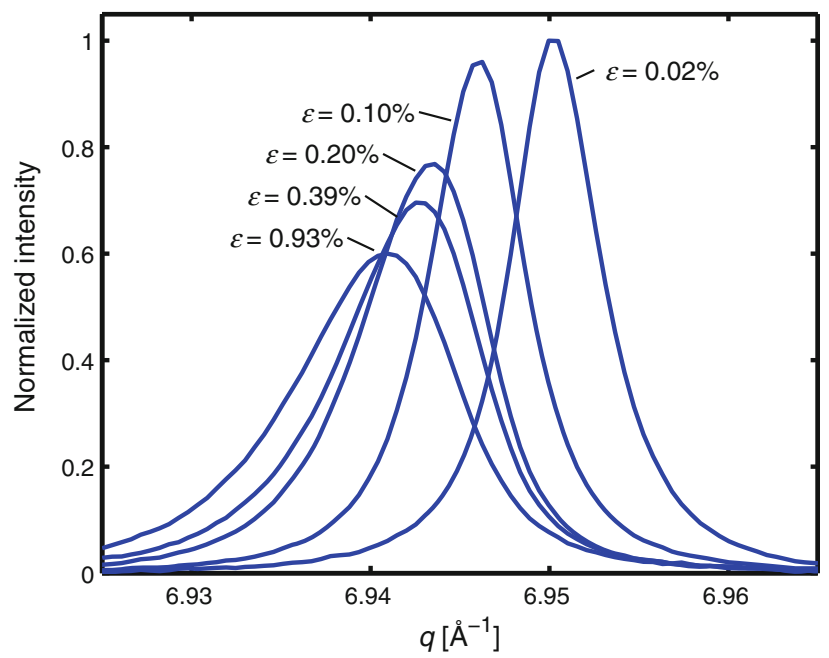

Fig. 8. Radial profiles from a grain embedded in a sample predeformed to a tensile strain of $5 \%$ obtained during in situ tensile deformation after an orthogonal strain path change $\left(55^{\circ}\right.$ between the tensile axes). The strain levels indicated refer to the macroscopic strain following the $5 \%$ prestrain. study the evolution of the dislocation structure in larger detail. The changes between the individual maps are only gradual, without any signs of drastic changes in the morphology. An example of a peak splitting into two peaks after a strain of $1.17 \%$ is indicated by an arrow.

\section{Radial X-Ray Peak Profiles and Asymmetry}

Additional insight in both regimes can be gained from analyzing the complementary radial profile of the 400 reflection from the entire grain. Figure 8 presents the radial profiles after different strains following the orthogonal strain path change.

The shift of the profiles with increasing strain is a consequence of the increasing applied load: As the diffraction vector of the selected 400 reflection is close to the tensile axis, the developing tensile stresses cause an increase in the related lattice spacing $d_{400}$. According to the diffraction condition $q=2 \pi / d$, the radial profiles shift to smaller values of the diffraction vector $q$ with the shift $\Delta q=-\varepsilon q$ directly determined by the elastic strain $\varepsilon$ along the diffraction vector.

With increasing macroscopic strain, an increased broadening of the profiles can be observed due to the increasing defect content. Most importantly, however, the formation of an asymmetry of the peak profile with the intensity decaying more slowly for smaller diffraction angles can be noticed.

Such an occurrence of asymmetric profiles during tensile deformation has been discovered earlier. ${ }^{26}$ They have been rationalized by the composite-like structure of dislocation structures ${ }^{27}$ consisting of hard dislocation-rich boundaries separating dislocation-free subgrains ${ }^{28}$ : With increasing tensile load, the softer subgrains will yield first, while dislocations cannot penetrate dislocation boundaries. Dislocations accumulated at the interface generate long-range elastic stresses acting in the same sense (tensile stresses) as the applied load in the dislocation walls and in the opposite sense (compressive back stresses) in the softer subgrains. Such stresses along the tensile direction cause elastic strains depending on the angle $\psi$ with the tensile direction and Poisson's ratio $v$

$$
\begin{aligned}
\varepsilon_{\psi} & =\varepsilon_{\| \mid} \cos ^{2} \psi+\varepsilon_{\perp} \sin ^{2} \psi=\left(\cos ^{2} \psi-v \sin ^{2} \psi\right) \varepsilon_{\|} \\
& =\left(1-(1+v) \sin ^{2} \psi\right) \frac{\sigma_{\|}}{E}
\end{aligned}
$$

The observed x-ray peak profiles are a superposition of partial profiles from dislocation walls and subgrains. Due to their differences in elastic strains, these subprofiles are shifted with respect to each other causing an asymmetry of the resulting radial profile.

The asymmetry of the x-ray peak profile is quantified here by the difference between the radial position of the maximum intensity and the average position of the entire radial profile. Figure 9 


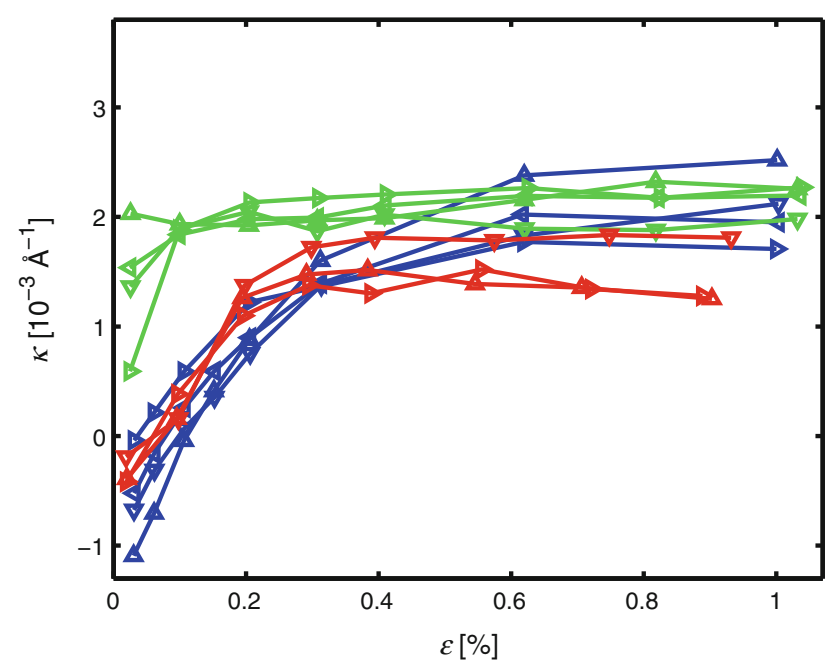

Fig. 9. Asymmetry following a strain path change in dependence on the macroscopic strain during reloading in tension after predeformation to a tensile strain of $5 \%$ with an angle of $0^{\circ}$ (green), $55^{\circ}$ (red), and $90^{\circ}$ (blue) between the tensile axes.

summarizes the asymmetry of peak profiles for different 400 reflections obtained for different grains and different strain path changes. Reflections from grains from specimens deformed without any strain path change show a positive asymmetry from the beginning of the reloading remaining almost constant in the investigated strain interval.

As a point of reference, Fig. 9 includes also the asymmetries observed for grains from specimens experiencing a change in the tensile direction by $90^{\circ} .^{29}$ In this case, the chosen diffraction vector close to the tensile direction of the in situ deformation is perpendicular to the tensile direction of the predeformation. Due to the Poisson effect, compressive stresses of subgrains along the original tensile direction will cause tensile elastic strains along the diffraction vector (side case, $\varepsilon_{90^{\circ}}=-v \varepsilon_{\|}$) and, hence, a negative asymmetry. ${ }^{29}$ When loading along the new tensile direction, back stresses along the new loading direction develop in the subgrains, leading to compressive strains along the new tensile direction and a reversal of the asymmetry.

Reflections from grains which experience a change in tensile direction of $55^{\circ}$ are initially quite symmetric and show only small asymmetries at all. The diffraction vector forms an angle of about $55^{\circ}$ with the tensile direction of the predeformation. As a consequence of the Poisson effect, stresses developing along the tensile direction will cause only minor strains along this particular direction (with $v=0.34$ for copper)

$$
\varepsilon_{55^{\circ}}=\frac{1-2 v}{3} \varepsilon_{\|} \approx 0.11 \varepsilon_{\|}
$$

Even if significant elastic strains develop along the tensile direction, almost no asymmetry can be

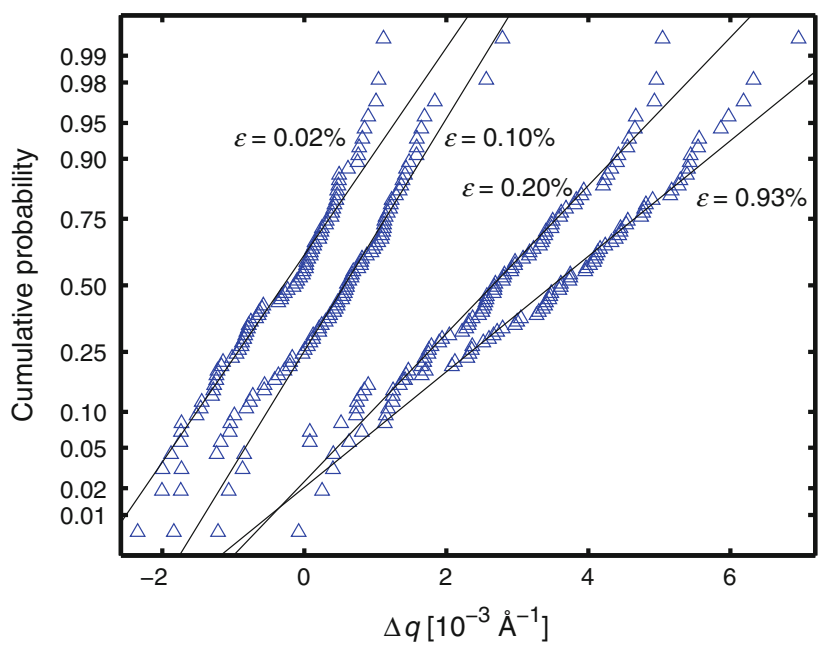

Fig. 10. Normal probability plot of the radial position of individual high-intensity peaks from the same grain embedded in a sample predeformed to a tensile strain of $5 \%$ obtained during in situ tensile deformation after an orthogonal strain path change $\left(55^{\circ}\right.$ between the tensile axes). The strain levels indicated refer to the macroscopic strain following the $5 \%$ prestrain.

expected for the profiles of reflections corresponding to diffraction vectors along this direction.

When loading the specimen along the new direction, i.e., with an orthogonal strain path change, elastic stresses are building up accordingly and a positive asymmetry is developed. From Fig. 9, it is obvious that dominant changes in the asymmetry occur during the initial straining, i.e., the microplastic regime. After $0.3 \%$ strain, the asymmetry has reached the value of the deformation without strain path change and remains unaltered further on.

\section{Behavior of Individual Subgrains}

In each of the azimuthal maps after an orthogonal strain path change of Fig. 6, subgrains are identified by their unique azimuthal positions. For the 80 largest identified subgrains, the corresponding radial positions with respect to the average of the grains are presented in a normal probability plot in Fig. 10. At each strain level, the data can be approximated reasonable by a straight line indicating that the radial positions of the subgrains are Gaussian distributed (in accordance with expectations ${ }^{28}$ ). For the predeformed sample, the radial positions of all subgrains are close to the average position of the grain, which is expected, as for this particular direction nearly no asymmetry is observed and the difference between the position of the maximum and the average is small. Nevertheless, a significant variation between the stresses of the individual subgrains exists.

With increasing strain, the radial positions of the subgrains show more and more positive values with 
respect to the average of the grain corresponding to the development of compressive backward strains as expected from the composite model. As observed earlier for unidirectional deformation, ${ }^{17,22}$ the radial positions of the high-intensity peaks gather in the vicinity of the radial position of the maximum intensity, not around the average value of the radial profile. Already for a macroscopic strain of $0.20 \%$, the average backward strain has reached a substantial positive value which does not change significantly with further deformation. During the microplastic regime, mechanical reloading of the subgrain structure occurs and a pronounced asymmetry of the radial profiles develops accordingly.

\section{CONCLUSIONS}

By investigating the reloading behavior of predeformed specimen with and without strain path changes a microplastic regime has been identified dominating the transient behavior during the first $0.2 \%$ to $0.3 \%$ of plastic strain. During this regime, the morphology of the subgrain structure (as identifiable in azimuthal maps of selected reflections) is not altered and the number and orientation of the individual subgrains are conserved. The developing of a substantial asymmetry of the radial profile (or reversal of an existing asymmetry) in strain path change experiments indicates a collective redistribution of the internal stresses during the microplastic regime to comply with the new deformation conditions. After the redistribution of internal stresses has established the proper load transfer according to the new deformation conditions, the subgrain structure evolves differently. The individual subgrains are no longer preserved; they disappear entirely or split apparently into several subgrains.

During the microplastic regime, only subgrains were yielding and deforming plastically. After having developed the proper stress state in the subgrain structure, both hard dislocation boundaries and soft subgrains deform plastically by moving dislocations. With the onset of macroscopic yielding and plastic deformation of the entire grain, the dislocation structure starts to reorganize by formation of new subgrains.

\section{ACKNOWLEDGEMENTS}

This work was funded by the Danish National Research Foundation via the Center for Fundamental Research: Metal Structures in Four Dimensions, by the ERC advanced grant "Diffraction based Transmission X-ray Microscopy", and by the Danish Natural Science Research Council via Danscatt. Use of the Advanced Photon Source was supported by the U.S. Department of Energy, Office of Science, Basic Energy Sciences under contract No. DE-AC0206CH11357. We are grateful to L. Lorentzen for preparing the samples and F. Adrian for assistance with the mechanical tests.

\section{OPEN ACCESS}

This article is distributed under the terms of the Creative Commons Attribution License which permits any use, distribution, and reproduction in any medium, provided the original author(s) and the source are credited.

\section{REFERENCES}

1. J. Gil Sevillano, Plastic Deformation and Fracture of Materials, ed. H. Mughrabi (Weinheim, Germany: Wiley VCH, 1993), p. 19.

2. U. Essmann, Phys. Status Sol. 3, 932 (1963).

3. J.W. Steeds, Proc. R. Soc. Lond. A 292, 343 (1966).

4. E. Göttler, Philos. Mag. 28, 1057 (1973).

5. D. Knoesen and S. Kritzinger, Acta Metall. 30, 1219 (1982).

6. X. Huang, Scripta Mater. 38, 1697 (1998).

7. M. Wilkens, Phys. Status Sol. (A) 2, 359 (1970).

8. M.A. Krivoglaz, X-Ray and Neutron Diffraction in Nonideal Crystals (Berlin, Germany: Springer Verlag, 1996), p. 1.

9. D. Breuer, P. Klimanek, and W. Pantleon, J. Appl. Cryst. 33, 1284 (2000).

10. T. Ungár, Mater. Sci. Eng. A 309-310, 14 (2001).

11. R. Kuzel, Z. Kristallogr. 222, 136 (2007).

12. S.V. Raj and G.M. Pharr, Mater. Sci. Eng. 81, 217 (1986).

13. B.C. Larson, W. Yang, G.E. Ice, J.D. Budai, and J.Z. Tischler, Nature 415, 887 (2002).

14. L.E. Levine, B.C. Larson, W. Yang, M.E. Kassner, J.Z. Tischler, A. Delos-Reyes, R.J. Fields, and W. Liu, Nat. Mater. 5, 619 (2006).

15. L.E. Levine, P. Geantil, B.C. Larson, J.Z. Tischler, M.E. Kassner, W.J. Liu, M.R. Stoudt, and F. Tavazza, Acta Mater. 59, 5803 (2011).

16. B. Jakobsen, H.F. Poulsen, U. Lienert, J. Almer, S.D. Shastri, H.O. Sørensen, C. Gundlach, and W. Pantleon, Science 312,889 (2006).

17. B. Jakobsen, H.F. Poulsen, U. Lienert, and W. Pantleon, Acta Mater. 55, 3421 (2007).

18. J.H. Schmitt, J.V. Fernandes, J.J. Gracio, and M.F. Vieria, Mater. Sci. Eng. A A147, 143 (1991).

19. N.A. Sakharova and J.V. Fernandes, Mater. Sci. Forum 514-516, 897 (2006).

20. J.H. Schmitt, E. Aernoudt, and B. Baudelet, Mater. Sci. Eng. 75, 13 (1985).

21. S.D. Shastri, J. Synchrotron Rad. 11, 150 (2004).

22. B. Jakobsen, U. Lienert, J. Almer, H.F. Poulsen, and W. Pantleon, Mater. Sci. Eng. A 483-484, 641 (2008).

23. B. Jakobsen, H.F. Poulsen, U. Lienert, J. Bernier, C. Gundlach, and W. Pantleon, Phys. Status Sol. (A) 206, 21 (2009).

24. C. Wejdemann, U. Lienert, H.B. Nielsen, and W. Pantleon: Proc. 31st Risø Intern. Symp. Mater. Sci.: Challenges in Materials Science and Possibilities in $3 D$ and $4 D$ Characterization Techniques, ed. N. Hansen, D. Juul Jensen, S.F. Nielsen, H.F. Poulsen, and B. Ralph (Roskilde, Denmark: Risø DTU, 2010), p. 477.

25. C. Wejdemann, Evolution of Dislocation Structures Following a Change in Loading Conditions Studied by In Situ High Resolution Reciprocal Space Mapping (Ph.D. thesis Ris $\varnothing-\mathrm{PhD}-74(\mathrm{EN})$, Roskilde, Denmark: Ris $\emptyset$ DTU, 2011), p. 1.

26. T. Ungár, H. Mughrabi, D. Rönnpagel, and M. Wilkens, Acta Metall. 32, 333 (1984).

27. H. Mughrabi, Acta Metall. 31, 1367 (1983).

28. W. Pantleon, C. Wejdemann, U. Lienert, and H.F. Poulsen: Proc. 31st Risø Intern. Symp. Mater. Sci.; Challenges in Materials Science and Possibilities in 3Dnd $4 D$ Characterization Techniques, ed. N. Hansen, D. Juul Jensen, S.F. Nielsen, H.F. Poulsen, and B. Ralph (Ris $\emptyset$ DTU, Roskilde, 2010), p. 79.

29. C. Wejdemann, U. Lienert, and W. Pantleon, Scripta Mater. $62,794(2010)$. 